\title{
Study of Combined Method for Double Frequency Sensing of Liquid Precipitation
}

\author{
Yevgeniy Belov, Stanislav Khomenko, Grygoriy Klopov, Anna Linkova*, Hennadii Rudnev, \\ Oleg Voitovych
}

Radar department, A. Ya. Usikov Institute for Radiophysics and Electronics of NAS of Ukraine, Kharkov, 61085, Ukraine

*Corresponding Author:annlinkova@mail.ru

Copyright (C) 2013 Horizon Research Publishing All rights reserved.

\begin{abstract}
The development of combined double frequency method of radar rain sensing and preliminary results of the experimental study of the method are presented. As it was shown the experimental study is in good agreement with calculations and confirms the main conclusions of the theoretical considerations.
\end{abstract}

Keywords Radar Cross Section, Differential Radar Cross-Section, Signal Attenuation, Rain Intensity

\section{Introduction}

Development of radar methods for measurement of rain parameters is of great interest for agriculture, municipal services and for scientific research in climatology, atmosphere physics etc [1].

At present the mono frequency methods [2,3] are widely used which are based on the correlation between rain intensity $I(\mathrm{~mm} / \mathrm{h})$ and radar reflectivity $Z\left(\mathrm{~mm}^{6} / \mathrm{m}^{3}\right)$ in the form $Z=A I^{B}$. However the coefficients $A$ and $B$ depend on different conditions (precipitation origin etc. [3]) and vary in wide range that decreases accuracy of such methods.

That is why the double frequency sensing $[4,5]$ is of great interest to increase reliability of the rain measurements. In this case the inverse task is defined relatively to parameters of drop size distribution (microstructure parameters). The integral characteristics (intensity, water content etc.) are calculated on the base of measured drop size distribution that is one of the advantages of such methods which are now widely used in satellite sensing $[6,7]$.

The double frequency sensing is successfully realized for two-parameter distributions for example Marshall-Palmer, Lows-Parson distributions etc. [8]. However they are not able to describe rain structure in the area of small and large drops [9]. At present Gamma distribution [9] of particles sizes is used in the most cases as a rain model. But it is impossible to determine all three distribution parameters by measurements at two frequencies. That is why the different approaches $[4,5,8,9]$ were studied to solve inverse task by use of additional correlation between Gamma distribution parameters. Particularly it was proposed $[10,11]$ to use data of contact measurements of dependences of gamma distribution parameters on rain intensity [12] which were obtained by statistical averaging of numerous measurements and so they can be characterized by high reliability.

The goal of the present work is verification of the proposed method by comparison of experimental data of double frequency radar and rain gauge contact measurements.

\section{Algorithm of Data Processing for Double Frequency Radar}

Double frequency sensing of rains is based on measurement of differential value of received power $P_{D}\left(\lambda_{1}, \lambda_{2}\right)=P_{R}\left(\lambda_{1}\right) / P_{R}\left(\lambda_{2}\right)$ at two wavelengths $\lambda_{1}, \lambda_{2}$ and calculation of differential radar cross section (DRCS) $\sigma_{D}\left(\lambda_{1}, \lambda_{2}\right)$ as ratio [11,13] of specific radar cross section (SRCS) $\sigma_{0}\left(\lambda_{1,2}\right)$ :

$$
\sigma_{D}\left(\lambda_{1}, \lambda_{2}\right)=\frac{\sigma_{0}\left(\lambda_{1}\right)}{\sigma_{0}\left(\lambda_{2}\right)}=\frac{P_{D}\left(\lambda_{1}, \lambda_{2}\right)}{C_{r}\left(\lambda_{1}, \lambda_{2}\right) K_{D}\left(\lambda_{1}, \lambda_{2}, R\right)}
$$

where $C_{r}=\frac{P_{T}\left(\lambda_{1}\right)}{P_{T}\left(\lambda_{2}\right)} \frac{G_{A}^{2}\left(\lambda_{1}\right) \lambda_{1}^{2}}{G_{A}^{2}\left(\lambda_{2}\right) \lambda_{2}^{2}} \frac{V_{e f}\left(\lambda_{1}\right)}{V_{e f}\left(\lambda_{2}\right)}$ is radar constant; $P_{T}$ is transmitter power; $G_{A}$ is antenna gain; $V_{e f}$ is effective scattering volume; $K_{D}\left(\lambda_{1}, \lambda_{2}, R\right)=\frac{K\left(\lambda_{1}\right)}{K\left(\lambda_{2}\right)}$ is differential value of attenuation coefficient $K\left(\lambda_{1,2}\right)[11,14] ; R$ is distance to the scattering volume (Fig. 1 ); $\sigma_{0}$ is specific value $\operatorname{RCS}[13,15]$ :

$$
\sigma_{0}(\lambda)=N_{T} \int_{0}^{\infty} \sigma_{p}(D, \lambda) F(D) d D
$$

where $\sigma_{p}(D, \lambda)$ is radar cross sections of one drop $\left(\mathrm{mm}^{2}\right)$ $[15,16] ; F(D)$ is probability density of drop size distribution; $D$ is drop diameter;

Gamma distribution is widely used for description of 
liquid precipitation [9]

$$
N(D)=N_{T} F(D)=N_{0} D^{\alpha} \exp \left(-\frac{D}{\beta}\right)
$$

where $N_{T}$ is drop concentration; $\alpha, \beta, N_{0}$ are distribution parameters, where $\quad N_{0}=N_{T} / \Gamma(\alpha+1) \beta^{\alpha+1} \quad$; $\Gamma(\alpha+1)=\int_{0}^{\infty} d t t^{\alpha} e^{-t}$ is Gamma function. In common case microstructure parameters depend on distance $R$, but such dependence is down hereinafter.

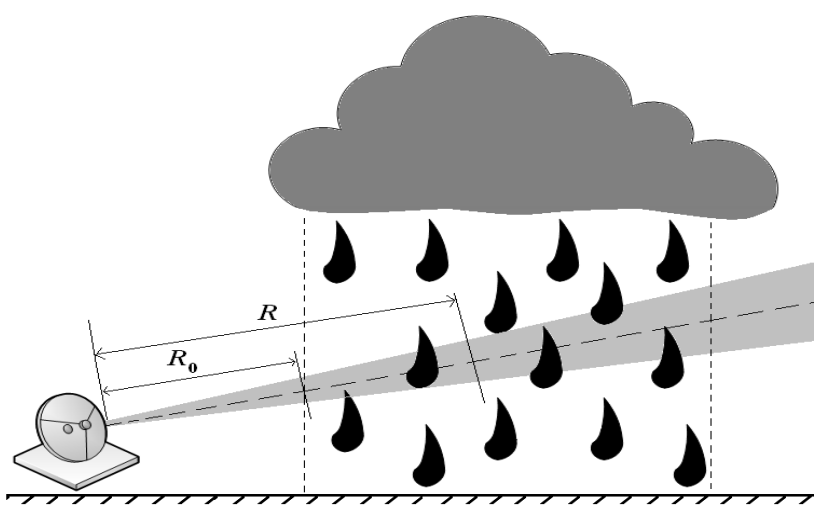

Figure 1. Radar sensing of rain zone

As it was shown in [13], the best results are achieved for double frequency sensing at the wavelengths $\lambda_{1}=8 \mathrm{~mm}$ and $\lambda_{2}=3.2 \mathrm{~cm}$. However for $\lambda=8 \mathrm{~mm}$ large drops in the drop size distribution correspond to the resonance scattering $\rho=\frac{\pi D}{\lambda_{1}} \sqrt{|\dot{\varepsilon}|} \geq 1$, where $\dot{\varepsilon}$ is complex permittivity of water.

That is why the rigorous solution of diffraction problem of electromagnetic waves by dielectric sphere (Mie theory [15]) is used for calculation of attenuation and radar cross sections.

Using expressions (1)-(8) the following equation can be obtained

$$
\frac{\sigma_{0}\left(\alpha, \beta, \lambda_{1}\right)}{\sigma_{0}\left(\alpha, \beta, \lambda_{2}\right)} K_{D}\left(\lambda_{1}, \lambda_{2}, R\right)=\frac{P_{D}\left(\lambda_{1}, \lambda_{2}\right)}{C_{r}\left(\lambda_{1}, \lambda_{2}\right)}
$$

which contains three unknown microstructure parameters. For them calculation a new approach was proposed in the works $[10,11]$ based on using experimental data of contact measurements of correlation between Gamma distribution parameters and rain intensity [12] $\alpha(I), \beta(I)$ :

$$
\alpha=a I^{-b}, \beta=c I^{d}, \mathrm{~mm}
$$

where $a=3.8 ; \mathrm{b}=0.42 ; c=0.148$ and $d=0.38$. Using (5) the correlation between two distribution parameters can be obtained:

$$
\beta=c\left(\frac{a}{\alpha}\right)^{\frac{d}{b}}
$$

Moreover the a priori information about scattering volume of rain is used $[10,11]$ as initial conditions for solution of expression (4). At that two kind of initial conditions can be: $\rightarrow$ radar is placed outside of rain zone at the distance R0 from the front border of rain. In this case attenuation is absent on the left of rain zone $K_{D}\left(R_{0}\right) \equiv 1$;

$\rightarrow$ radar is placed inside of rain zone and initial values of microstructure parameters can be found by contact measurements.

Let us to consider remote sensing by the radar with high range resolution $\Delta R$, when rain microstructure parameters are uniform within one resolution cell. At that rain zone of length $L$ contains $M$ resolution cells $1 \leq m \leq M=\operatorname{Int}\left(\frac{L}{\Delta R}\right)$, and distance for them is equal to $R_{m}=R_{0}+(m-1) \Delta R$ (Fig. 2 ). Then for initial conditions of first type the differential coefficient of attenuation for $m$-th cell can be written as product of attenuation coefficients in the previous cells $q \leq m$

$$
K_{D}\left(R_{m}\right)=\prod_{q=1}^{m} \exp \left\{-\Phi_{q}\right\}
$$

where $q$ is order number of resolution cell and expression for exponent is follow

$$
\Phi_{q}=0.002 \Delta R N_{T_{q}} \int_{0}^{\infty} d D F\left(D, \alpha_{q}, \beta_{q}\right) \Delta \sigma\left(D, \lambda_{1}, \lambda_{2}\right),
$$

where $\Delta \sigma=\left[\sigma_{A T}\left(D, \lambda_{1}\right)-\sigma_{A T}\left(D, \lambda_{2}\right)\right] ; \alpha_{q}=\alpha\left(R_{q}\right) ; \beta_{q}=$ $\beta\left(R_{q}\right) ; N_{T q}=N T\left(R_{q}\right)$.

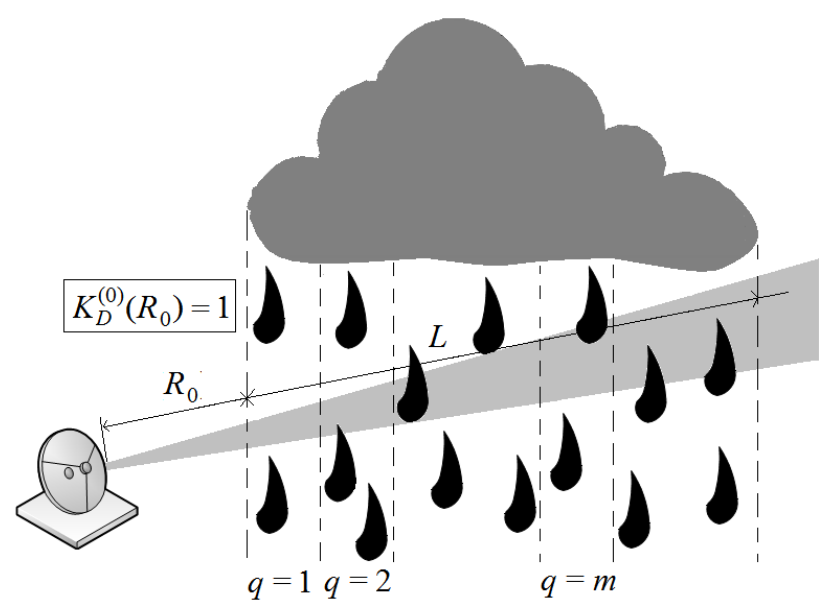

Figure 2. Scheme of rain radar sensing

Values of microstructure parameters for the first resolution cell $\alpha_{1}$ and $\beta_{1}$ can be found from solution of inverse task (4)-(8) for loss-free medium, where $K_{D}\left(R_{0}\right) \equiv 1$ :

$$
\left\{\begin{array}{l}
\sigma_{D}\left(\alpha_{1}, \beta_{1}\right)=\frac{P_{D}\left(\lambda_{1}, \lambda_{2}\right)}{C_{r}\left(\lambda_{1}, \lambda_{2}\right)} \\
\beta_{1}=c\left(\frac{a}{\alpha_{1}}\right)^{\frac{d}{b}}
\end{array}\right.
$$


And distribution parameter $N_{01}$ - from solution of following expression

$$
N_{01}=\frac{\sigma_{0}\left(R_{0}, \lambda_{2}\right) \beta_{1}^{-\left(\alpha_{1}+1\right)}}{\Gamma\left(\alpha_{1}+1\right) \int_{0}^{\infty} d D \sigma_{p}\left(D, \lambda_{2}\right) F\left(D, \alpha_{1}, \beta_{1}\right)}
$$

To define values of microstructure parameters more exactly the expressions (9), (10) should be considered as initial conditions for the next iterations. In common case expression for iterations of order $p \geq 1$ is written in the form

$$
\begin{gathered}
\sigma_{D}\left(\alpha_{1}^{(p-1)}, \beta_{1}^{(p-1)}\right) K_{D}\left(\alpha_{1}^{(p-1)}, \beta_{1}^{(p-1)}, N_{0_{1}}^{(p-1)}\right)= \\
=\frac{P_{D}\left(\lambda_{1}, \lambda_{2}\right)}{C_{r}\left(\lambda_{1}, \lambda_{2}\right)} ; \\
\beta_{1}^{(p)}=c\left(\frac{a}{\alpha_{1}^{(p)}}\right)^{\frac{d}{b}} \\
N_{01}^{(p)}=\frac{\sigma_{0}\left(\lambda_{2}\right)\left[\beta_{1}^{(p)}\right]^{-\left(\alpha_{1}^{(p)}+1\right)}}{\Gamma\left(\alpha_{1}^{(p)}+1\right) \int_{0}^{\infty} d D \sigma_{p}\left(D, \lambda_{2}\right) F\left(D, \alpha_{1}^{(p)}, \beta_{1}^{(p)}\right)}
\end{gathered}
$$
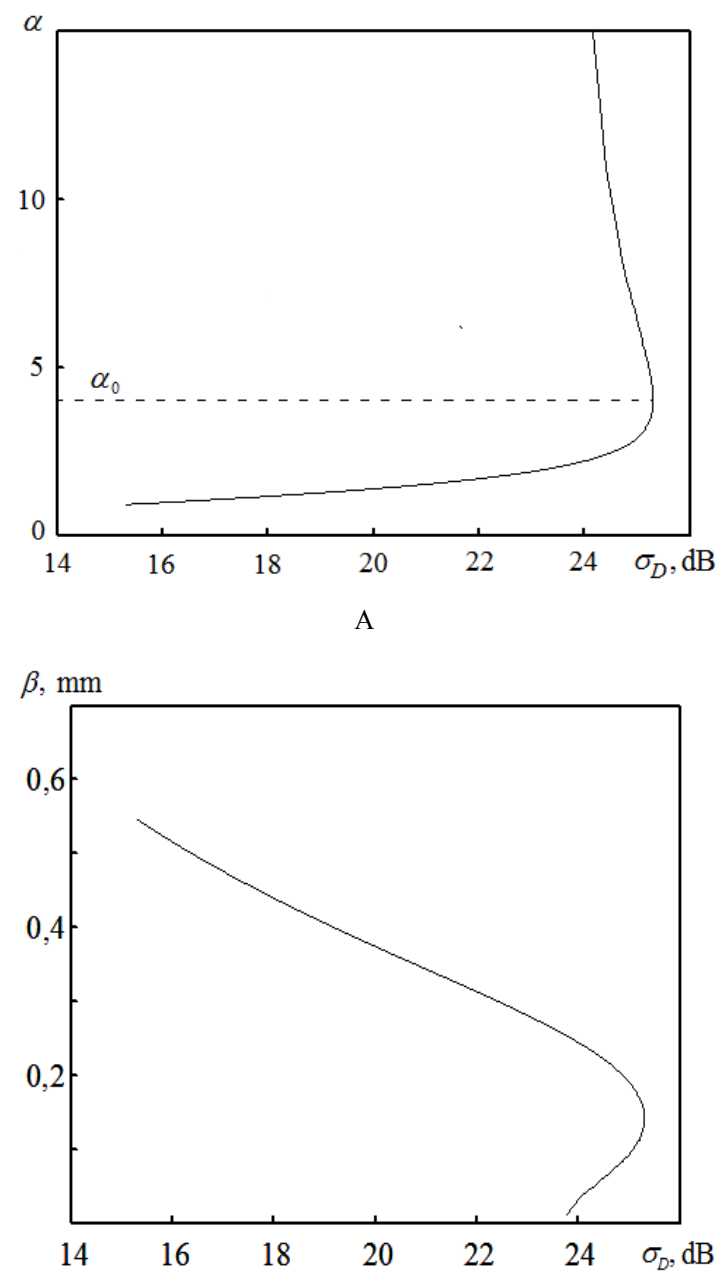

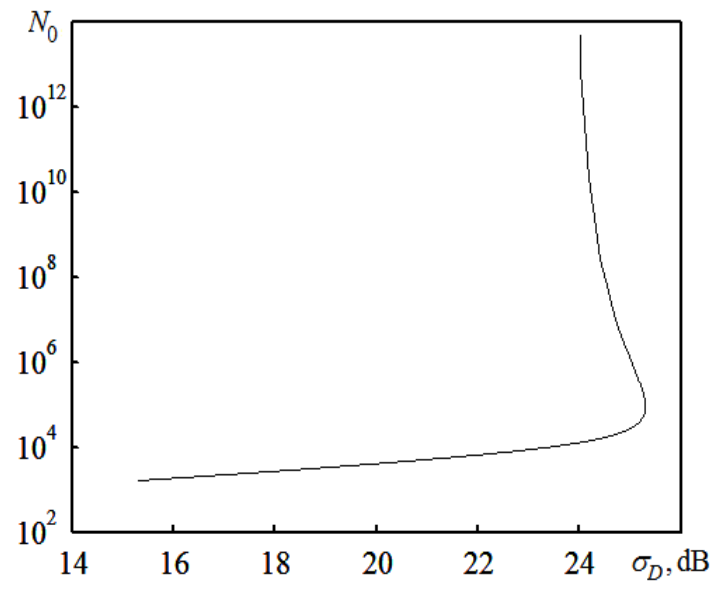

$\mathrm{C}$

Figure 3. Dependence of microstructure parameters on DRCS: A $-\alpha ; \mathrm{B}-$ $\beta ; \mathrm{C}-\mathrm{N} 0$

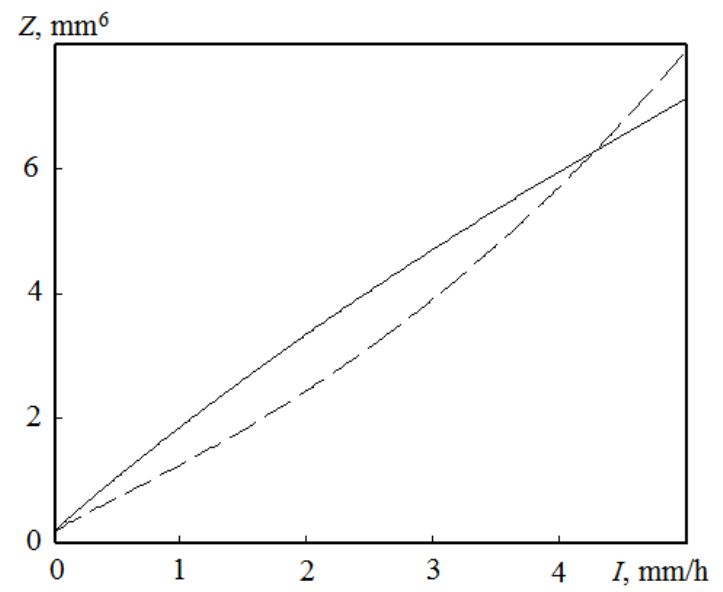

Figure 4. Dependence of radar reflectivity on rain intensity: solid line $-\lambda$ $=8.2 \mathrm{~mm}$; dashed line $-\lambda=3.2 \mathrm{~cm}$

Calculated values of microstructure parameters for the first resolution cell are considered as initial values for next cell. It permits to sequentially apply described algorithm for the rest of resolution cells $2 \leq m \leq M$, and obtain profile of microstructure parameters. However there is double-valued calculation of microstructure parameters by means of DRCS (Fig. 3) because of nonlinear dependences of radar reflectivities $Z$ on rain intensity at the wavelengths $\lambda_{1}=8$ $\mathrm{mm}$ and $\lambda_{2}=3.2 \mathrm{~cm}$ (Fig. 4). In this case one value of DRCS corresponds to two values of distribution parameter.

To disambiguate the dependences of microstructure parameters on SRCS at two wavelengths are used, which have monotonic character instead of DRCS (Fig. 5). It allows to present system of inequalities for choice of one of the dependence branch (Fig. 4)

$$
\begin{cases}\alpha \leq \alpha_{0} \text { for } \sigma_{0}\left(\lambda_{1}\right) \leq \sigma_{01}, & \sigma_{0}\left(\lambda_{2}\right) \leq \sigma_{02} ; \\ \alpha>\alpha_{0} \text { for } \sigma_{0}\left(\lambda_{1}\right)>\sigma_{01}, & \sigma_{0}\left(\lambda_{2}\right)>\sigma_{02},\end{cases}
$$

where $\alpha_{0}=4.05, \sigma_{01}=11.94 \mathrm{~mm}^{2} / \mathrm{m}^{3}, \sigma_{02}=0.035 \mathrm{~mm}^{2} / \mathrm{m}^{3}$, and their sense is illustrated by Fig. 5 . 


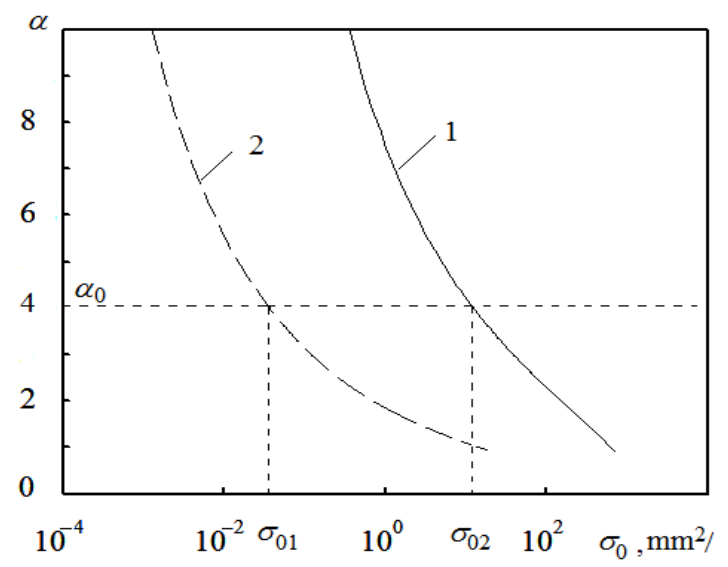

Figure 5. Dependence of distribution parameter $\alpha$ on SRCS: $1-\lambda=8.2$ $\mathrm{mm} ; 2-\lambda=3.2 \mathrm{~cm}$

Thereby the calculated values of microstructure parameters $\alpha, \beta$ and $N_{0}$ permit to define integral parameters of rain, including drop concentration, rain intensity and water content.

Convergence to the true value of calculating parameter is important property of iterative scheme. Dependence of rain intensity on radar range resolution $\Delta R$ calculated by described iterative procedure (solid lines) are presented in Fig. 6 for rain models with uniform profile of intensity (dashed lines). As it can be seen for rain intensity $I \geq 10$ $\mathrm{mm} / \mathrm{h}$ inaccuracy of calculations strongly increases for radar resolution $\Delta R \geq 600 \mathrm{~m}$ because assumption of uniform distribution of microstructure parameters within resolution cell (7) fulfils worse for larger values of intensity.

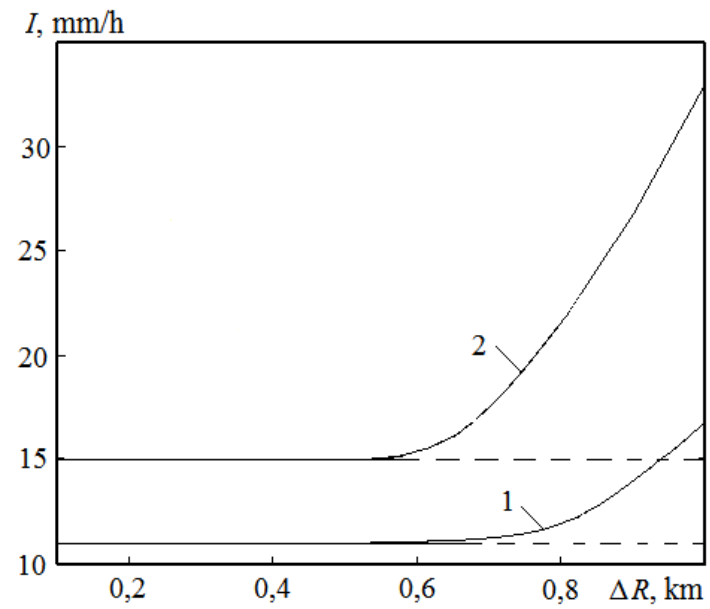

Figure 6. Convergence of iterative scheme for rain models with uniform profile of intensity: $1-\mathrm{I}=11 \mathrm{~mm} / \mathrm{h} ; 2-\mathrm{I}=15 \mathrm{~mm} / \mathrm{h}$

However for typical radars range resolution is usual $\Delta R \cong 150 \mathrm{~m}$ therefore application of proposed iterative procedure provides high accuracy even for rains of intensity $I \cong 15 \mathrm{~mm} / \mathrm{h}$ (Fig.7: solid lines - model profiles, dots reconstructed profiles). Number of iterations in the worst case is not more than five. At that applicability of described method is limited by measurement accuracy of rain SRCS. Taking into account accuracy of radar measurement $\pm 1 \mathrm{~dB}$ then the maximal value of rain intensity which can be measured by proposed method is not more $20 \mathrm{~mm} / \mathrm{h}$.

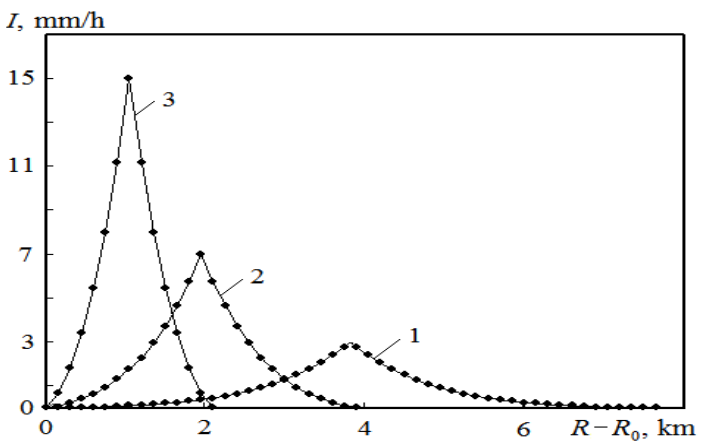

Figure 7. Reconstruction of profile of rain intensity: $1-\operatorname{Imax}=3 \mathrm{~mm} / \mathrm{h} ; 2$ $-\operatorname{Imax}=7 \mathrm{~mm} / \mathrm{h} ; 3-\operatorname{Imax}=15 \mathrm{~mm} / \mathrm{h}$

\section{Equipment for Experimental Study of Proposed Method}

Radar complex (Fig. 8) was used for experimental study based on pulsed incoherent weather radar of type MRL-1 [17] and fast-acting rain gauge. At that additional radar blocks were developed (Fig. 8B): unit for calibration of radar sensitivity (CU), TV-viewing device (TV) for optical identification of observed objects, control unit of antenna positioning (AC), interface unit (IU) for data exchange between radar and PC and the radar control. PC provides control of radar antenna and calibration of receiver sensitivity jointly with inbuilt power meter of transmitter and developed software permit to measure absolute values of reflected signal power.

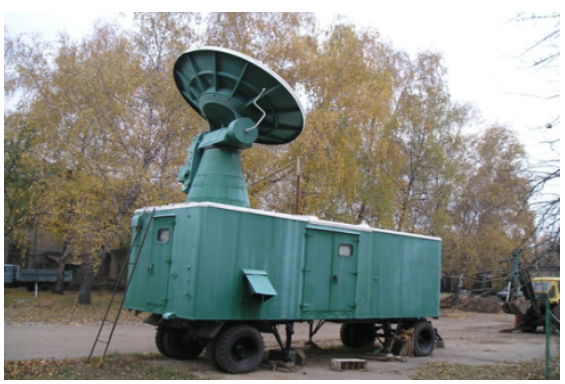

A

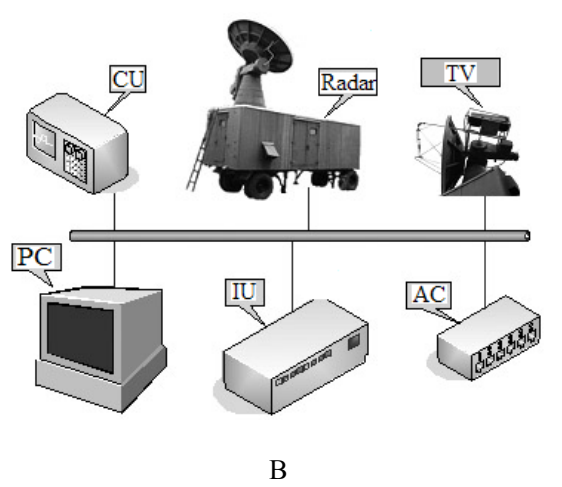

Figure 8. Radar complex: A - general view; B - block diagram 
At that nonlinearity of amplitude characteristic of receiver is compensated in the range $45 \mathrm{~dB}$. It provides measurement of radar reflectivity of observed objects with accuracy not worse than $\pm 1.0 \mathrm{~dB}$ taking into account of calibration of antenna gain and loss in the antenna-feeder device.

The preliminary signal processing and data transmission are performed between pulses of the radar with repetition period $3.33 \mathrm{~ms}(300 \mathrm{~Hz})$. The time interval within which the reflected signals are received is about $250 \mu$ s (maximal range $37.5 \mathrm{~km}$ ), the interval of preliminary signal processing is 230 $\mu \mathrm{s}$ and the data transmission into $\mathrm{PC}$ is performed within $2853 \mu \mathrm{s}$.

Interface unit forms pulse driver of radar transmitter-receiver, controls antenna position and provides analog-digital conversion of received signals.

The observation time of angle sector $0^{\circ} \div 90^{\circ}$ is about $\approx 77$ sec (antenna scanning pitch $\approx 1^{\circ}$, number of range cells 250 ). The main characteristics of double frequency radar are presented in Table 1.

Table 1. Radar parameters

\begin{tabular}{|c|c|c|}
\hline Parameter & Channel 1 & Channel 2 \\
\hline Wavelength $\lambda, \mathrm{mm}$ & 8.2 & 32 \\
\hline Pulsed power $P_{T}, \mathrm{kWt}$ & 70 & 250 \\
\hline Antenna gain $G_{A}, \mathrm{~dB}$ & 58 & 46 \\
\hline Antenna beam width, degree & 0.22 & 0.7 \\
\hline Range resolution, $\mathrm{m}$ & 75 & 150 \\
\hline
\end{tabular}

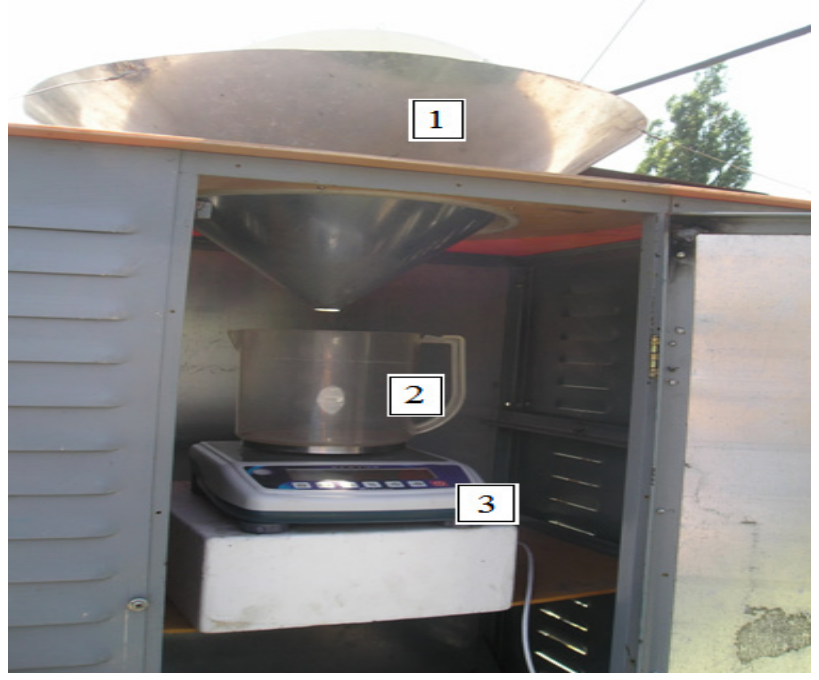

Figure 9. Rain gauge: 1 - funnel, 2 - vessel, 3 - electronic balance

To verify the data of remote sensing the high-performance rain gauge was developed on the base of electronic balance (Fig. 4). The water drops through the entry funnel 1 fill the plastic vessel 2, which is placed on the electronic balance platform 3. The software for the balance permits to calculate time dependence of rain intensity $(\mathrm{mm} / \mathrm{h})$ and rainfall amount $Q(\mathrm{~mm})$ accordingly to the expressions

$$
I(t)=\frac{3,6 \cdot 10^{6}}{\rho_{w} S_{0}} \frac{\Delta M(t)}{\Delta t}, \quad Q=\frac{\Delta t}{3,6 \cdot 10^{3}} \sum_{n=1}^{N} I\left(t_{n}\right),
$$

where $\rho_{w}$ is water density, $\mathrm{g} / \mathrm{mm}^{3} ; S_{0}=\pi r^{2}$ is square of entry port of the funnel; $r$ is radius of entry port of the funnel $(2 r=500 \mathrm{~mm}), \Delta M(t)$ is increment of water mass per time period $\Delta t(\mathrm{~s})$.

\section{Results of Experimental Study}

The goal of the experimental study is verification of the proposed method by comparison of experimental data obtained by double frequency radar and rain gauge. For this the mode of vertical sensing (antenna was vertically oriented) for radar was used and rain gauge was located straight near the radar (Fig. 10). Averaging time of data for rain gauge is 6 $\mathrm{sec}$ and for radar $-0.86 \mathrm{sec}$ (256 samples). At that the time of data updating for radar is $2 \mathrm{sec}$.

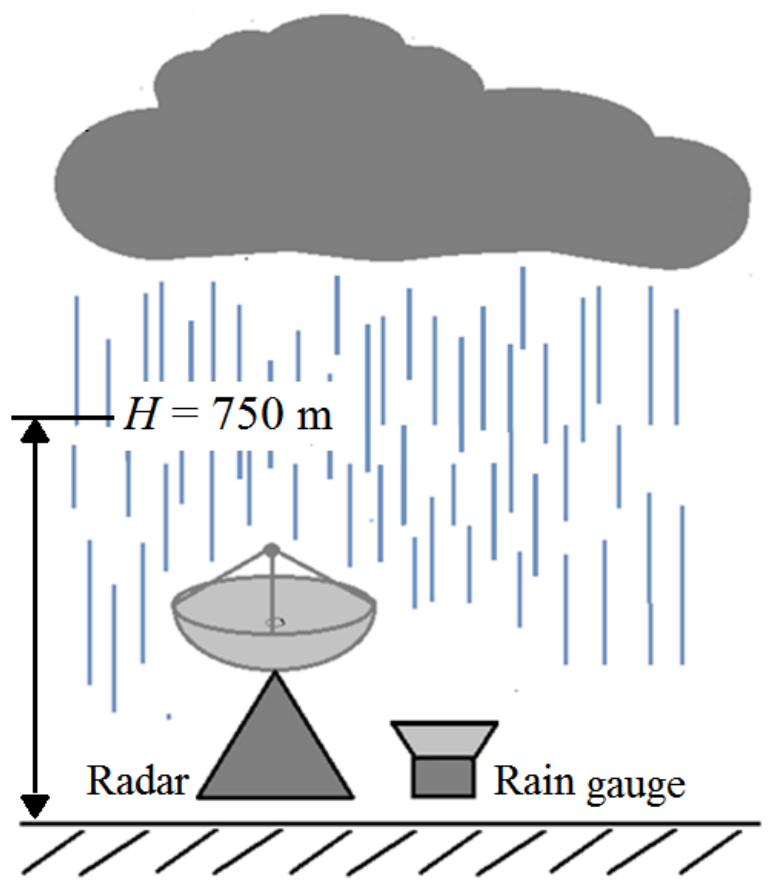

Figure 10. Scheme of experiment performance

In a common case comparison of remote and contact data is quite difficult task because it is necessary to provide performance of measurements for the same spatial area of rain. The lack of lateral drift of rain drops during the fall is the main condition for this. In additional radar and rain gauge data are spaced in time at indeterminate interval due to unknown speed of drop fall. That is why cross-correlation function of rain intensity measured by radar $I_{r}(t)$ and rain gauge $I_{r g}(t)$ was calculated

$$
K(\tau)=\int_{0}^{\infty} d t I_{r g}(t) I_{r}(t-\tau)
$$

and the time of drop fall $\tau_{r g}$ corresponds to the maximum of 
correlation coefficient $K\left(\tau_{r g}\right)=K_{\max }$.

An example of radar image of one of the observed rain with uniform distribution of intensity is presented in Fig. 11 which corresponds to the practically full absence of wind (mean wind speed $\bar{V}_{w}<0.5 \mathrm{~m} / \mathrm{sec}$ ) and vertical drop fall.

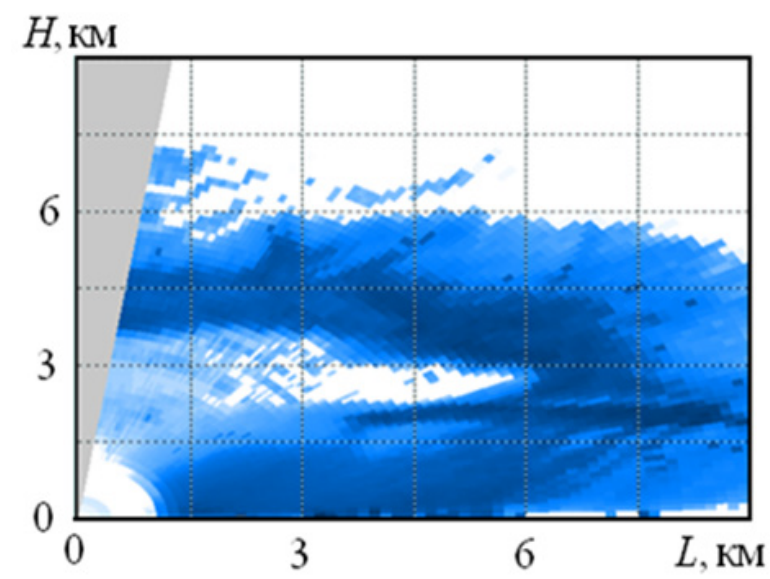

Figure 11. Radar image of rain

Experimental results of intensity measurements are shown in Fig. 12 for the resolution cell placed at the height $H=750$ $\mathrm{m}$. The both dependences are plotted in the real time scale and the time delay between the radar and rain gauge data occurs because fall time from the height $750 \mathrm{~m}$ to ground surface, where the contact measurements was performed. Calculation of maximum of correlation function (14) $K\left(\tau_{r g}\right) \cong 0.8$ permitted to define delay between radar and rain gauge data $\tau_{r g} \cong 114 \mathrm{sec}$, which corresponds to mean speed of rainfall $6.8 \mathrm{~m} / \mathrm{sec}$.

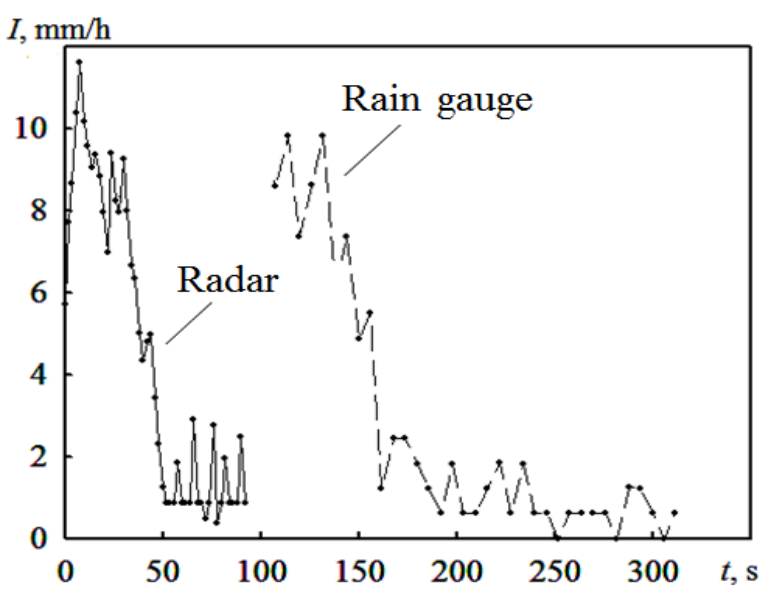

Figure 12. Comparison of remote sensing and contact measurements of rain intensity

Agreement of radar and rain gauge data in each moment of time should not be expected because the described algorithm of double frequency sensing is built on the average data of contact measurements [12]. But it can be seen quality similar character of dependences. Moreover remote and contact measurements (Table 2) averaged for time $20 \div 30 \mathrm{sec}$ differ not more than $\delta_{I}=2\left|\frac{\bar{I}_{r}-\bar{I}_{r g}}{\bar{I}_{r}+\bar{I}_{r g}}\right| \cdot 100 \% \leq 15 \%\left(\bar{I}_{r}, \bar{I}_{r g}\right.$ are mean values of rain intensity obtained by radar and rain gauge respectively).

Table 2. Comparison of averaged rain intensity

\begin{tabular}{|c|c|c|c|}
\hline № & $I_{r}, \mathrm{~mm} / \mathrm{h}$ & $I_{r g}, \mathrm{~mm} / \mathrm{h}$ & Inaccuracy, \% \\
\hline 1 & 8.76 & 8.34 & 4.9 \\
\hline 2 & 4.11 & 4.73 & 14.1 \\
\hline 3 & 1.39 & 1.37 & 1.5 \\
\hline 4 & 3.94 & 3.79 & 3.9 \\
\hline
\end{tabular}

Proposed double frequency method is formulated accordingly to the microstructure rain parameters so it allows to define not only rain intensity but and other parameters including, modal diameter $D_{\text {mod }}$, drop concentration $N_{T}\left(\mathrm{~m}^{-3}\right)$, appearance of drop size distribution $F(D)$ and water content $W\left(\mathrm{~g} / \mathrm{m}^{3}\right)$.

In Fig. 13 the time structure of microstructure parameters of rain shown in Fig. 12 is presented. As it can be seen the microstructure parameters essentially vary so different drop size distribution can correspond to the same intensity. Particularly this explains applicability limits of the mono frequency methods based on $Z-I$ relation.

Also the saturation regions of dependences should be noted which can be explained by dependence of drop concentration on rain intensity (Fig. 14) accordingly to $[12,18]$. At the beginning the rain intensity increases because of increase of drop concentration and then - due to increase of drop size.

Obtained data were used for calculation (18)-(20) of drop concentration $N_{T}$ (Fig. 15) and water content $W$ (Fig. 16). At that the maximal values of concentration and water content correspond to the time of maximal rain intensity (Fig. 12). Because of strong time dependence of calculated rain parameters their mean values are of great interest (Table 3). It should be noted that in spite of different rain intensity $(I=$ $1 \div 9 \mathrm{~mm} / \mathrm{h}$ ) modal diameter does not have essential variation as it also can be seen from calculated drop size distribution on the base of Table 3 (Fig. 17).

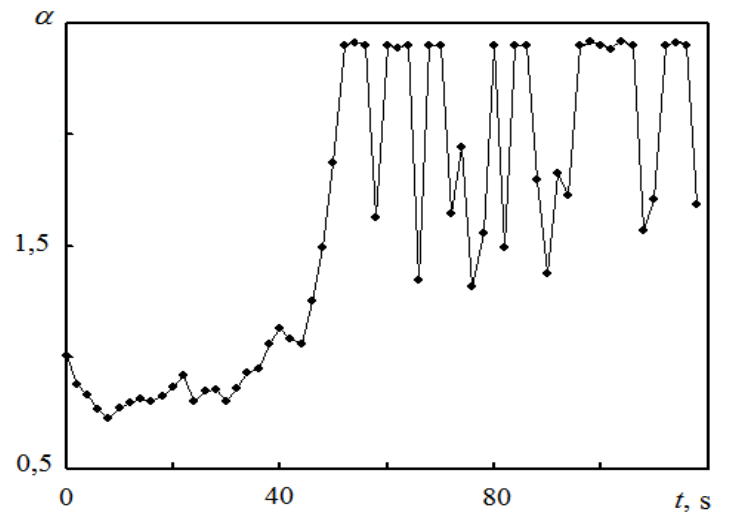




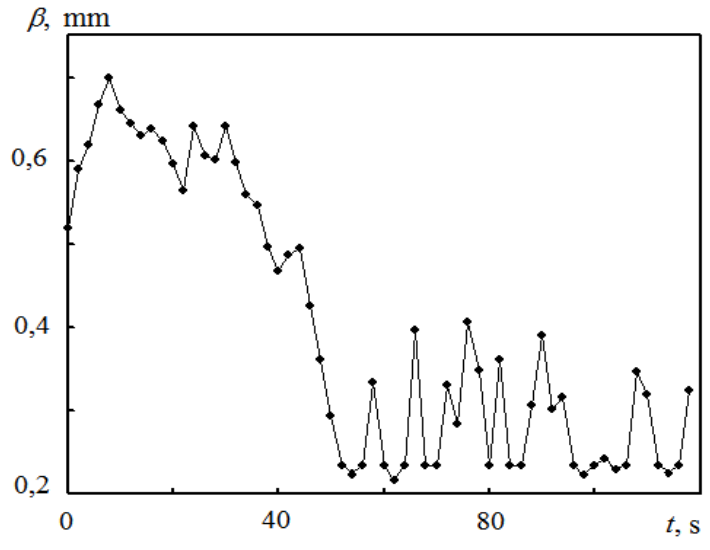

B

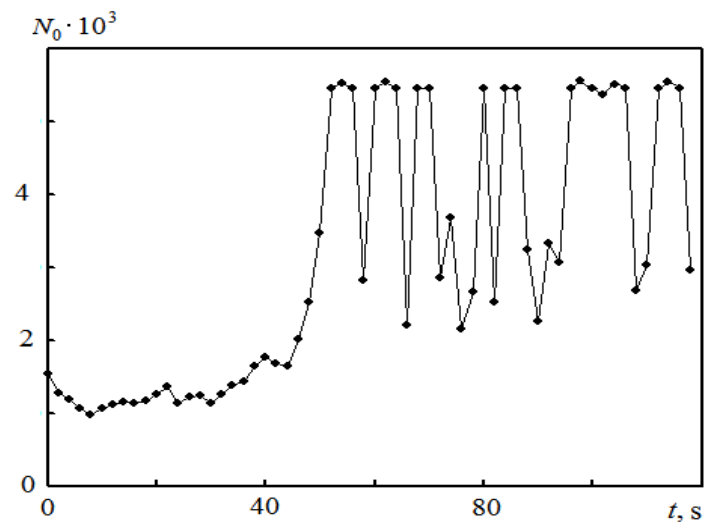

C

Figure 13. Time structure of microstructure parameters of rain: $A-\alpha(t)$; $\mathrm{B}-\beta(\mathrm{t}) ; \mathrm{C}-\mathrm{N} 0(\mathrm{t})$

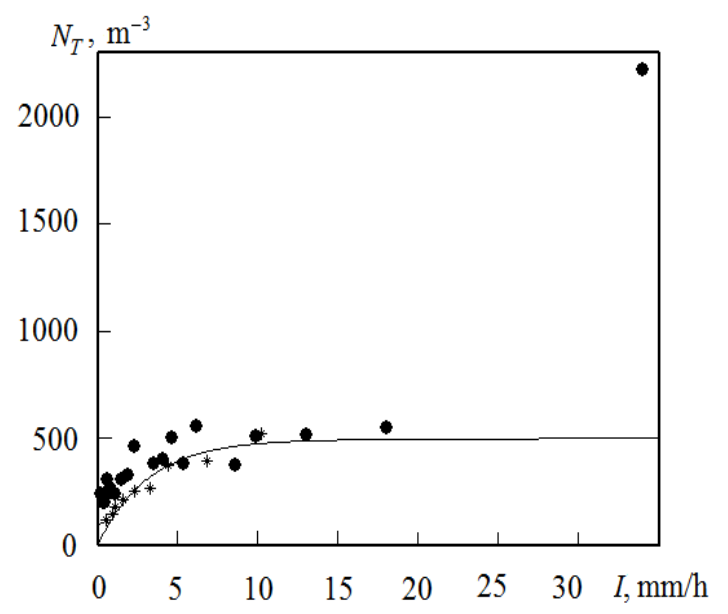

Figure 14. Dependence of drop concentration on rain intensity: solid line is approximation of data $[12,18]$

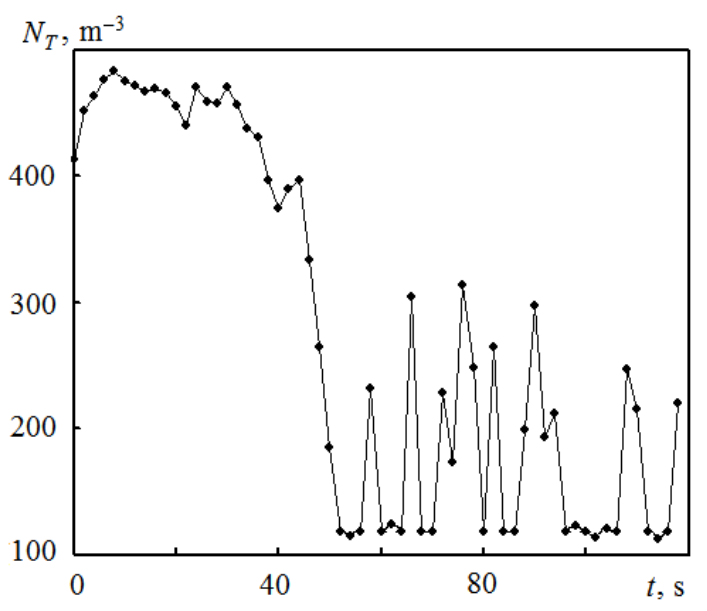

Figure 15. Time structure of drop concentration for rain shown in Fig. 12

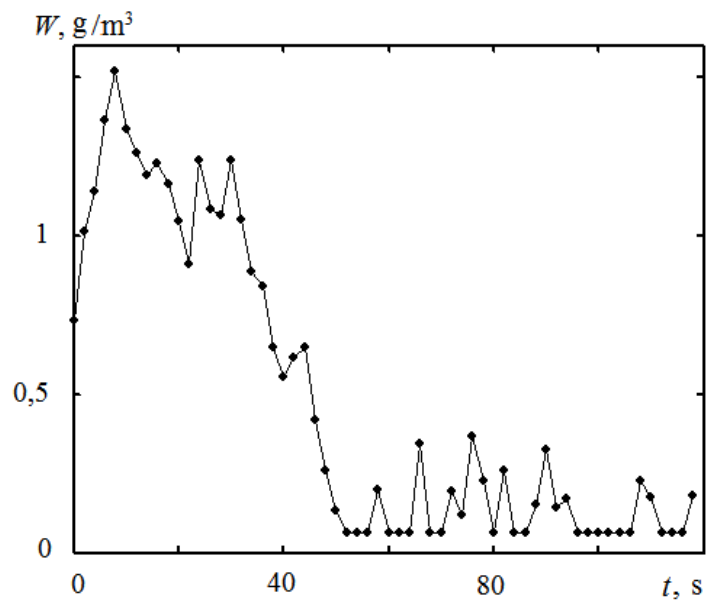

Figure 16. Time structure of water content for rain shown in Fig. 12

Table 3. Mean values of rain parameters

\begin{tabular}{|c|c|c|c|c|}
\hline \multirow{2}{*}{ Parameter } & \multicolumn{4}{|c|}{ Number of experiment } \\
\cline { 2 - 5 } & 1 & 2 & 3 & 4 \\
\hline $\bar{\alpha}$ & 0.83 & 1.63 & 2 & 1.59 \\
\hline $\bar{\beta}, \mathrm{mm}$ & 0.62 & 0.38 & 0.28 & 0.4 \\
\hline $\bar{N}_{0}$ & 1188 & 3180 & 4240 & 3100 \\
\hline $\bar{N}_{T}, \mathrm{~m}^{-3}$ & 461 & 270 & 174 & 279 \\
\hline $\bar{D}_{\text {mod }}, \mathrm{mm}$ & 0.51 & 0.54 & 0.55 & 0.54 \\
\hline
\end{tabular}




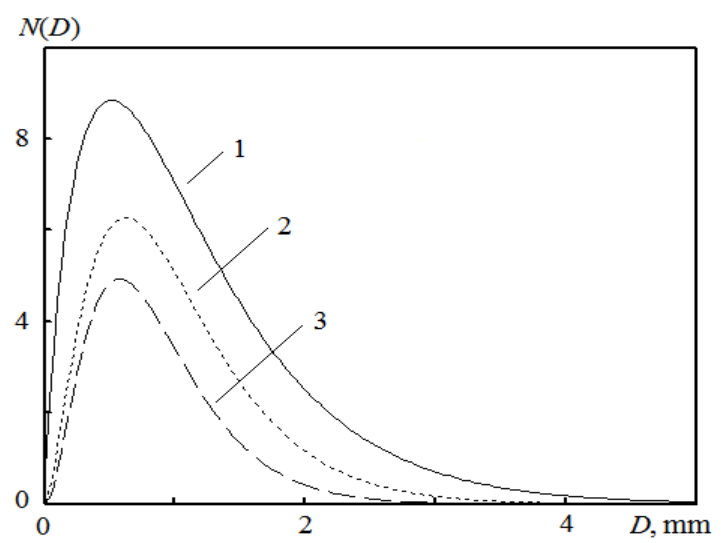

Figure 17. Drop size distribution for observed rains: $1-\mathrm{I}=8.8 \mathrm{~mm} / \mathrm{h} ; 2-$ $\mathrm{I}=4.1 \mathrm{~mm} / \mathrm{h} ; 3-\mathrm{I}=1.4 \mathrm{~mm} / \mathrm{h}$

At the same time comparison of radar and rain gauge data as it was mentioned above is not always possible, particularly in the case of presence of cross wind. Radar image of nonuniform rain is presented in Fig.18 obtained for strong cross wind в (mean speed $\bar{V}_{w} \cong 8 \mathrm{~m} / \mathrm{s}$ ).

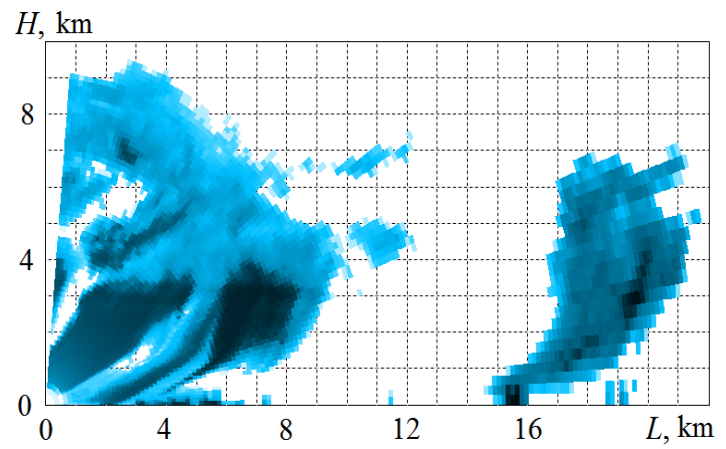

Figure 18. Radar image of nonuniform rain

At that comparison of remote (Fig. 19) and contact (Fig. 20) data showed their essential disagreement (maximal value of intensity $I_{r} \cong 18 \mathrm{~mm} / \mathrm{h}$ and $I_{r g} \cong 9 \mathrm{~mm} / \mathrm{h}$ respectively). So it is impossible to compare radar and rain gauge data in the case of strong cross wind.

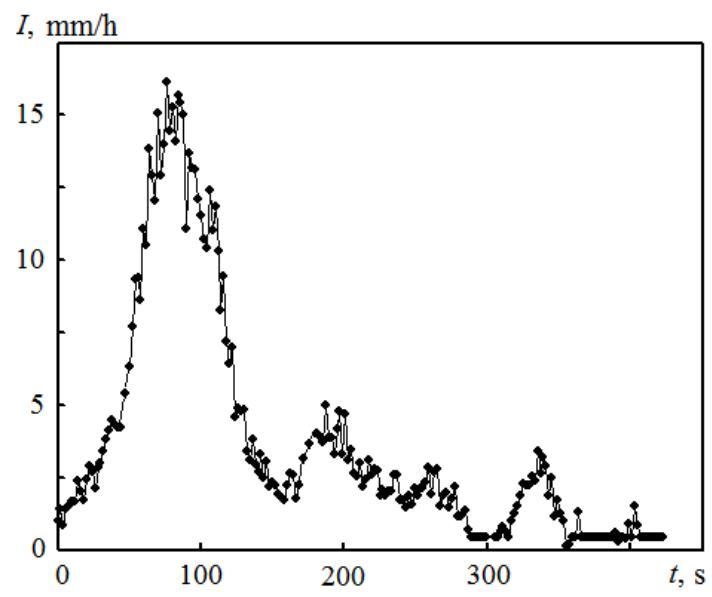

Figure 19. Time dependence of rain intensity measured by radar in the presence of strong cross wind

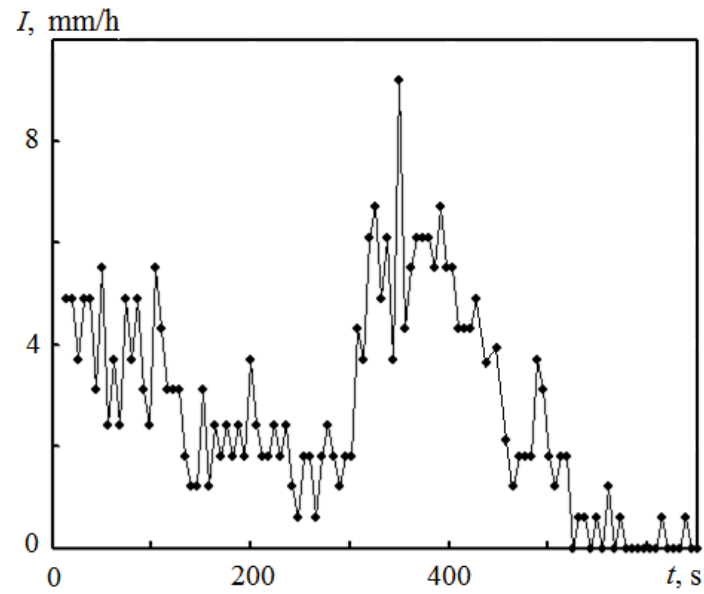

Figure 20. Time dependence of rain intensity measured by rain gauge in the presence of strong cross wind

\section{Conclusions}

1. Experimental complex for double frequency sensing of rains was developed and built based on weather radar MRL-1 and fast acting rain gauge.

2. Approach for comparison of radar and rain gauge data was developed to estimate efficiency of proposed double frequency method.

3. Experimental study showed availability of proposed algorithm for data processing of double frequency sensing of rains. However for accuracy estimation of the method it is necessary to perform additional experiments in the wide range of rain intensity and in different seasons.

\section{REFERENCES}

[1] R. S. Scorer. Aerodynamics of environment, Mir, Moscow, 1980.

[2] Atlas D., Radar in Meteorology, Academic Press, Inc., 1967, $194 \mathrm{p}$.

[3] R. Jameson, A.B. Kostinski. Spurious power-law relations among rainfall and radar parameters, Q.J.R. Meteorol. Soc. Vol. 128, 2045-2058, 2002.

[4] S. J. Munchak. Retrieval of Raindrop Size Distribution from Simulated Dual-Frequency Radar Measurements, J. Appl. Meteor. and Climatology, Vol.47, 223-239, 2008.

[5] R. Menenghini, L. Liao. On the equivalence of dual-wavelength and dual-polarization equations for estimations of the raindrop size distribution, J. Atmos. and oceanic technology, Vol. 24, 806-820, 2007.

[6] C.R. Rose, V. Chandrasekar. A GPM Dual-Frequency Retrieval Algorithm: DSD Profile-Optimization Method, J. of atmospheric and oceanic technology, Vol. 23, 1372-1383, 2006.

[7] R. Menenghini, T. Kozu, W. C. Boncyck A study of rain 
estimation methods from space using dual-wavelength radar measurement at near-nadir incidence over ocean, J. Atmos. Oceanic Technol, Vol. 9, 364-382, 1992.

[8] A. Seifert. On the shape-slope relation of drop size istributions in convective rain, J. Appl. Meteor, No. 44, 1146-1151.

[9] Z. S. Haddad, S. L. Durden, E. Im. Parameterizing the raindrop distribution, J. Appl. Meteor, Vol.35, 3-13, 1996.

[10] A. Linkova, G. Khlopov, S. Khomenko, O. Voitovych, Double frequency sounding of liquid precipitation, 11th International Radar Symposium (IRS'2010), Vol.1, 322-325, 2010.

[11] A . Linkova. Use of microstructure parameters for data processing of double frequency measurement of rain intensity, J. RadioPhysics and Electronics, IRE NASU, Vol.2 (16), No.1, 33-38, 2011.

[12] I. V. Litvinov. Structure of atmosphere precipitation,
Gidrometeoizdat, Leningrad, 1974.

[13] M. Cherniakov, G. Khlopov, A. Linkova, O. Voitovych, Use of double frequency radar for measurements of rain parameter profile, 12th International Radar Symposium (IRS'2011), 250-255, 2011.

[14] Attenuation of laser radiation in hydrometeors / Edited by M. A. Kolosov, Nauka, Moskow, 1977.

[15] H. C. van de Hulst. Light scattering by small particles, John Wiley \& Sons, New York, 1957.

[16] G. Khlopov, A. Linkova, O. Voitovych. Double Frequency Profiling of Rain Parameters, J. RadioPhysics and Electronics, Vol.16, No.3, 51-60, 2011.

[17] V. D. Stepanenko. Radar in meteorology, Gidromrtroizdat, Leningrad, 1973.

[18] V. N. Kelkar. Size distribution of raindrops, III, Indian J. Meteorol. Geophys, Vol.12, No.4, 553, 1961. 